(Aus dem pharmakologischen Institute der Universität Breslau.)

\title{
Zur Frage nach dem Sauerstoffbedürfnisse des Froschnerven.
} Von

Dr. K. H. Baas, Assistent.

H. v. Baeyer ${ }^{1}$ ) hat in einer unter Verworn angestellten Arbeit: „Das Sauerstoffbedürfnis des Nerven" festgestellt, dass infolge von Sauerstoffentziehung durch indifferente Gase der markhaltige Nerv gelähmt werde und bei Zutritt von Sauerstoff sich wieder erhole. Diese für die Physiologie des Nerven so wichtige Thatsache schien den bisherigen Angaben und namentlich der allgemeinen Auffassung so wiỏersprechend, dass eine Nachprüfung der Thatsache dringend wünschenswert war. Deshalb habe ich mich dieser Aufgabe unterzogen.

In einigen Punkten bin ich von der Verworn'schen Versuchsanordnung abgewichen. Wenn, wie v. B a eyer fand, der Nerv bei Durchleitung von reinem, sauerstofffreiem Stickstoff erst nach 2 bis 15 Stunden unerregbar wird und bei Zuführung von reinem Sauerstoff in drei bis fünf Minuten oder, wie Fröhlich ${ }^{2}$ ), der ebenfalls unter Verworn die Untersuchungen B a eyer's aufnahm und bestätigte, schon nach einer Minute wieder erregbar wurde, so war anzunehmen, dass atmosphärische Luft statt reinen Sauerstoffs genommen werden konute. Gestlutzt auf die Untersuchungen von $\operatorname{Hermann}{ }^{3}$ ), der nachwies, dass der ausgeschnittene Frosehmuskel in wirklich reinen indifferenten Gasen und besonders im Vakuum ebenso lange, unter Umständen sogar länger erregbar bleibe als in der Luft, konnte ich eine Versuchsanordnung wählen, die wesentlich bequemer war als die Verw orn'sche.

1) Zeitschr. f. allgem. Physiol. Bd. 2 H. 1 S. 169.

2) Zeitschr. f. allgem. Physiol. Bd. 3 H. 1 S. 75.

3) Handbuch Bd. 2 S. 132. 
Als Nervmuskelpräparat dienten ebenso wie in den Versuchen von v. Baeyer die bis zum Knie freipräparierten Nervi ischiadici mit dem ganzen nicht abgehäuteten Beine, das unmittelbar über dem Knie amputiert war.

Das Nervmuskelpräparat wurde, in geeigneter Weise isoliert, an einem Stativ befestigt. Das so armierte Stativ stand in einer Glasschale, an der in zweckentsprechender Weise zwei biegsame Kabeldrähte mit Platinelektroden befestigt waren, so dass der Nerv in bequemer Weise über die Elektroden gebrückt und das ganze Stativ unter eine geräumige tubulierte Glasglocke gesetzt werden konnte. Der Abschluss des Glockenraumes gegen die umgebende Luft wurde durch destilliertes Wasser oder ausgekochtes Leitungswasser hergestellt. Das Nervmuskelpräparat wurde, ehe es unter die Glocke kam, mit physiologischer Kochsalzlösung oder Froschlymphe angefeuchtet. Ausserdem wurde die Innenwand der Glocke noch etwa zur Hälfte mit stark durchtränktem Fliesspapier bekleidet. Zur Erstickung wurde Wasserstoff benutzt, der aus reinem Zink und chemisch reiner Salzsäure dargestellt wurde. Zur Reinigung von etwa mitgerissener Salzsäure und Sättigung mit Feuchtigkeit wurde er zunächst durch eine Waschflasche mit destilliertem Wasser, dann durch Kalilauge, dann wieder durch destilliertes Wasser und zuletzt durch das Abschlusswasser unter die Glocke geleitet. Der Gummikorken der Glocke trug die Gasableitungsröhre, welche in eine Waschflasche mit Wasser leitete, um ein Eindringen von Luft zu verhindern, und zur besseren Kontrolle der Gasdurchleitung. Nach der Waschflasche mit Kalilauge wurde durch ein Y-förmiges Rohr das mit Luft gefüllte Gasometer angeschlossen. Durch Hähne und Klemmen konnte das Zuströmen der Gase reguliert oder unterbrochen werden. Zur Reizung diente ein durch zwei Daniell'sche Elemente armiertes Schlitteninduktorium. Als ein Erfolg kurzdauernder tetaniseher Reizung galt mir die minimalste Zuckung, die noch mit dem Auge wahrgenommen werden konnte. Der Rollenabstand von 180 wurde nicht überschritten, um Stromschleifen zu vermeiden. In der Regel durchströmten die Glocke, die etwa $2000 \mathrm{ccm}$ fasste, in der Minute 200 Gasblasen. In Anbetracht des grossen Rauminhaltes der Glocke und der langsamen Verdrängung des Wasserstoffs durch Luft, bei welcher der Partiardruck des Sauerstoffs nur äusserst langsam anwachsen konnte, war ich überrascht von der schnellen Wiederherstellung des Nerven, nämlich in durch- 
schnittlich fünf Minuten. Ich lasse hier das Protokoll meines zweiten Versuches folgen:

\begin{tabular}{|c|c|c|c|}
\hline Zeit, & $\begin{array}{l}\text { Rollen- } \\
\text { abstand }\end{array}$ & $\begin{array}{l}\text { Erfolg } \\
\text { Reizu }\end{array}$ & $\begin{array}{l}\text { der } \\
\text { ng }\end{array}$ \\
\hline 1135 & 600 & + & \\
\hline 1145 & 600 & + & \\
\hline 1200 & 600 & + & \\
\hline $12 \quad 15$ & 600 & + & \\
\hline $12 \quad 30$ & 600 & + & \\
\hline $12 \quad 45$ & 600 & + & \\
\hline 100 & 600 & + & \\
\hline 115 & 600 & + & \\
\hline 130 & 600 & + & \\
\hline 140 & 600 & + & \\
\hline 300 & 600 & + & \\
\hline 315 & 600 & + & sehr schwach \\
\hline $3 \quad 30$ & 600 & + & sehr schwach, Tetanus bei 400 \\
\hline 400 & 180 & 0 & $\begin{array}{l}\text { Wasserstoffzuleitung unter- } \\
\text { brochen und Luft durch- } \\
\text { geleitet }\end{array}$ \\
\hline $4 \quad 05$ & 600 & + & \\
\hline
\end{tabular}

Die Erregbarkeit des Nerven blieb also lange Zeit auf der Höhe, um dann ziemlich rasch abzusinken und schnell bei Zuleitung von Luft wieder anzusteigen. Ferner lässt sich das Ersticken und Wiederbeleben wiederholt an demselben Nerven nachweisen.

\begin{tabular}{|c|c|c|c|}
\hline Zeit, & $\begin{array}{l}\text { Rollen- } \\
\text { abstand }\end{array}$ & $\begin{array}{c}\text { Erfolg } \\
\text { Reizt }\end{array}$ & $\begin{array}{l}\text { der } \\
\text { ng }\end{array}$ \\
\hline 1135 & 600 & + & \\
\hline 1145 & 600 & + & \\
\hline $12 \quad 15$ & 600 & + & \\
\hline 1230 & 600 & + & \\
\hline 1245 & 600 & + & \\
\hline 100 & 600 & + & \\
\hline 115 & 600 & + & \\
\hline 130 & 600 & + & \\
\hline 140 & 600 & + & \\
\hline 300 & 600 & + & \\
\hline 315 & 600 & + & sebr schwach \\
\hline 330 & 600 & + & $\begin{array}{l}\text { sehr schwach, bei } 400 \text { starke } \\
\text { Reaktion }\end{array}$ \\
\hline $4 \quad 00$ & 180 & 0 & $\begin{array}{l}\text { Wasserstoffzuleitung unter- } \\
\text { brochen und Luft durch- } \\
\text { geleitet }\end{array}$ \\
\hline 405 & 600 & + & \\
\hline
\end{tabular}


Zur Frage nach dem Sauerstoffbedürfnisse des Froschnerven.

\begin{tabular}{|c|c|c|c|c|}
\hline \multicolumn{2}{|c|}{ Zeit } & $\begin{array}{l}\text { Rollen- } \\
\text { abstand }\end{array}$ & \multicolumn{2}{|c|}{$\begin{array}{l}\text { Erfolg der } \\
\text { Reizung }\end{array}$} \\
\hline 4 & 07 & Luft wieder abgestellt & and $w$ & eder Wasserstoff durchgeleitet \\
\hline 4 & 17 & 600 & + & \\
\hline 4 & 30 & 600 & + & \\
\hline 4 & 45 & 600 & + & \\
\hline 5 & 00 & 600 & + & \\
\hline 5 & 15 & 600 & + & \\
\hline 5 & 30 & 600 & + & \\
\hline 5 & 45 & 600 & + & \\
\hline 6 & 15 & 600 & + & \\
\hline 6 & 30 & 600 & + & \\
\hline 6 & 45 & 600 & + & \\
\hline 7 & 00 & 600 & + & sehr schwach \\
\hline 7 & 05 & 600 & + & $\begin{array}{l}\text { die ersten } 3 \text { Reizungen } \\
\text { schwach, } 4,5 \text { u. } 6 \text { erfolglos }\end{array}$ \\
\hline 7 & 10 & 600 & + & $\begin{array}{l}\text { die erste Reizung sehr } \\
\text { schwach, } 2,3 \text { u. } 4 \text { erfolglos }\end{array}$ \\
\hline 7 & 15 & 180 & 0 & \\
\hline 7 & 20 & 180 & 0 & $\begin{array}{l}\text { Wasserstoffzuleitung unter- } \\
\text { brochen und Luft eingeleitet }\end{array}$ \\
\hline 7 & 27 & 180 & 0 & \\
\hline 7 & 35 & 400 & + & \\
\hline 7 & 40 & 600 & + & \\
\hline
\end{tabular}

Sehr merkwürdig in diesem Versuche ist, dass der Nerv durch das Durchleiten von Luft während sieben Minuten, von 4 Uhr bis 4 Uhr 7 Minuten, wieder so hergestellt wurde, um trotz der darauffolgenden Wasserstoffdurchleitung drei Stunden und acht Minuten wieder erregbar zu bleiben, zumal wenn man in Betracht zieht, dass der schädliche Raum in der Glocke beinahe 1800 ccm betrug.

Ein Nervmuskelpräparat eines Sommerfrosches, bei dem zur Kontrolle unter sonst gleicher Versuchsanordnung nur Luft durch die Glocke geleitet wurde, blieb acht Stunden und fünf Minuten erregbar. Die zur Erstickung erforderliche Zeit der Gasdurchleitung schwankte in meinen Versuchen zwischen 1 Stunde 15 Minuten und 4 Stunden 25 Minuten bei Sommerfröschen im Juni und Juli. Im Winter dagegen zwischen etwa vier und acht Stunden.

War die Verworn'sche Anschauung über das Sauerstoffbedürfnis des Nerven, zu deren Gunsten auch unsere Versuche sprechen, richtig, so mussten auch mittels Gaspumpe und nachträglichen Einleitens von Luft dieselben Resultate sich erzielen lassen wie in unseren bisherigen Versuchen. Auf diesen Punkt hat 
schon v. Baeyer hingedeutet. Schon A. Ewald ${ }^{1}$ ) hatte im Jahre $186^{\circ} 9$, um über das Sauerstoffbedürfnis des Nerven ins Klare zu kommen, zwei Nervmuskelpräparate, das eine im Vakuum, das andere in Luft, verglichen und gefunden, dass bald das eine, bald das andere länger reagierte. Mit den Resultaten von Ewald sind weder die Verw orn'schen noch meine Ergebnisse vereinbar. Da wir in unseren Versuchen einen Febler nicht entdecken konnten, so musste der Fehler bei $\mathrm{E} w$ ald gesucht werden. Wie ich gezeigt, genügt schon ein äusserst geringer Partiardruck des Sauerstoffs, um den erstickten Nerven wieder erregbar zu machen. Es lag also nahe, anzunehmen, dass in den Ewald'schen Versuchen im Präparat und im angeblichen Vakuum wenn auch sehr geringe, doch ausreichende Mengen Sauerstoff vorhanden waren. Hat er doch nicht angegeben, dass er von Zeit zu Zeit das Evakuieren wiederholt habe. Unter Vermeidung eines solchen eventuellen Fehlers stellte ich die Versuche, wie folgt, an. Ich benutzte zum Erzeugen des Vakuums die Pflüger Geissler'sche Quecksilberluftpumpe und einen Glascylinder, an der einen Seite geschlossen und an der anderen mit eingeschliffenem Glashahn versehen. Seitlich waren zwei Platindrähte eingeschmolzen. Auf einem flachen Korken, der in die Rundung des Cylinders passte, wurde das Nervmuskelpräparat mit zwei Nadeln befestigt, und der Nerv über die Elektroden gelegt. Korken und Präparat wurden mit physiologischer Kochsalzlösung reichlich angefeuchtet, so dass in der Röhre noch freie Flüssigkeit vorhanden war, und darauf geachtet, dass der Korken die Platindrähte nicht berührte.

Versuch rom 6. Januar 1904.

\begin{tabular}{cccc}
\multicolumn{2}{c}{ Zeit } & $\begin{array}{c}\text { Rollen- } \\
\text { abstand }\end{array}$ & $\begin{array}{c}\text { Erfolg der } \\
\text { Reizung }\end{array}$ \\
10 & 00 & 520 & + Evakuation \\
10 & 10 & 640 & + \\
10 & 50 & 605 & + \\
11 & 00 & 550 & + \\
11 & 30 & 545 & + \\
12 & 00 & 545 & + \\
12 & 25 & 550 & + Nochmalige Evalkuation \\
12 & 40 & 565 & + \\
1 & 00 & 565 & + \\
3 & 00 & 510 & + \\
3 & 30 & 495 & +
\end{tabular}

1) Pflïger's Arch. Bd. 2 S. 142. 1869. 
Zur Frage nach dem Sauerstoffbedürfnisse des Froschnerven.

\begin{tabular}{cccc}
\multicolumn{2}{c}{ Zeit } & $\begin{array}{c}\text { Rollen- } \\
\text { abstand }\end{array}$ & $\begin{array}{c}\text { Erfolg der } \\
\text { Reizung }\end{array}$ \\
4 & 00 & 465 & + \\
4 & 30 & 180 & 0 \\
4 & 40 & 180 & 0 \\
4 & 50 & 520 & + \\
4 & 55 & 545 & + \\
5 & 15 & 555 & +
\end{tabular}

Fast jedesmal konnte ich sofort nach dem Evakuieren eine Steigerung der Erregbarkeit konstatieren, z. B. von Rollenabstand 520 auf 640 . Diese Erregbarkeitssteigerung dürte in Analogie zu setzen sein mit derjenigen, die sich beim Absterben des Nerven und bei schneller Wasserentziehung (Verdunstung) bemerkbar macht.

Es ist durch die Versuche von v. Baeyer, die von mir hiermit bestätigt worden sind, bewiesen, dass der Sauerstoff für die Thätigkeit des ausgeschnittenen Froschnerven notwendig ist, und wahrscheinlich gemacht, dass die Nervenfaser auch im normalen $\mathrm{Zu}$ stande an der Gewebsatmung entsprechenden Anteil nimmt. 